\title{
Promoção de saúde bucal, diminuição do medo e aumento do vínculo com pacientes pediátricos na sala de espera odontológica: um relato de experiência
}

\author{
Oral health promotion, decreased fear and increased bonding with pediatric patients in the \\ dental waiting room: an experience report
}
Promoción de la salud oral, disminución del miedo y mayor vinculación con pacientes pediátricos en la sala de espera dental: un informe de experiencia

Rafaella Bandeira de Melo Souza Cavalcanti ${ }^{1 *}$, Vitor Nascimento Goes ${ }^{1}$, Paula Lima Nogueira ${ }^{1}$, Quemuel Pereira da Silva ${ }^{1}$, Filipe de Oliveira Lima ${ }^{1}$, Rachel de Queiroz Ferreira Rodrigues ${ }^{1}$, Fátima Roneiva Alves Fonseca ${ }^{1}$, Maria Carolina Bandeira Macena ${ }^{1}$.

\section{RESUMO}

Objetivo: Relatar a experiência de acadêmicos que integraram a equipe do programa de extensão universitária nomeado como "Heróis do Sorriso", ligado ao curso de Odontologia em uma universidade pública do interior do estado da Paraíba, durante o desenvolvimento de ações com pacientes pediátricos na sala de espera da Clínica Escola da própria universidade. Relato de experiência: Atividades de caráter lúdico e interativo foram realizadas com crianças de 1 a 12 anos que estavam em tratamento odontológico nos turnos da manhã e tarde na Clínica Escola de Odontologia da faculdade, que fica localizada na cidade de Patos Paraíba, durante o ano de 2018. Uma das finalidades do projeto foi minimizar o medo do contato das crianças com o dentista, na tentativa de facilitar a aceitação do tratamento e o aumento do vínculo com a criança, além do incentivo ao engajamento no autocuidado e da promoção da própria saúde bucal. Considerações finais: Conclui-se que a vivência possibilitou aos estudantes de Odontologia construir uma forma inovadora de educação em saúde oral, baseando-se em práticas educativas e dinâmicas, o que contribuiu no sentido de vislumbrar um horizonte de atuação mais humanizado que supere as antigas práticas de cuidados pontuais e imediatistas.

Palavras-chave: Odontopediatria, Promoção da saúde, Higiene bucal, Humanização da assistência.

\begin{abstract}
Objective: To report the experience of academics who were part of the university extension program team named "Heroes of the Smile", linked to the Dentistry course at a public university in the interior of the state of Paraíba, during the development of actions with pediatric patients in the waiting for the School Clinic of the university itself. Experience report: Playful and interactive activities were carried out with children from 1 to 12 years old who were undergoing dental treatment in the morning and afternoon shifts at the Dental School Clinic located in the city of Patos - Paraíba, during the year 2018. One of the aims of the project was to minimize the fear of children's contact with the dentist in an attempt to facilitate the acceptance of treatment and increase the bond with the child, in addition to encouraging engagement in self-care and promoting oral health itself. Final considerations: It is concluded that the experience made it possible for Dentistry students to build an innovative form of oral health education, based on educational and dynamic practices, which contributed towards envisioning a more humanized performance horizon that surpasses the old ones punctual and immediate care practices.
\end{abstract}

Keywords: Pediatric dentistry, Health promotion, Oral hygiene, Humanization of assistance.

${ }^{1}$ Universidade Federal de Campina Grande (UFCG), Patos - PB.

*E-mail: rafaella.bm@hotmail.com

SUBMETIDO EM: 8/2020

ACEITO EM: 9/2020

PUBLICADO EM: 11/2020

REAS/EJCH | Vol.12(11) | e4991 | DOI: https://doi.org/10.25248/reas.e4991.2020 Página 1 de 6 


\section{RESUMEN}

Objetivo: Informar la experiencia de los académicos que formaron parte del equipo del programa de extensión universitaria llamado "Heróis do Sorriso", que está vinculado al curso de Odontología en una universidad pública del interior del estado de Paraíba, durante el desarrollo de acciones con pacientes pediátricos en la sala de espera de la clínica escolar de la universidad. Informe de experiência: Actividades lúdicas e interactivas se llevaron a cabo con niños de 1 a 12 años que estaban recibiendo tratamiento dental en los turnos de la mañana y la tarde en la clínica de la escuela de odontología ubicada en la ciudad de Patos Paraíba, durante 2018. Uno de los propósitos del proyecto era minimizar el miedo al contacto de los niños con el dentista en un intento de facilitar la aceptación del tratamiento y aumentar el vínculo con el niño, además de alentar la participación en el autocuidado y promover la salud bucal. Consideraciones finales: Se concluye que la experiencia hizo posible que los estudiantes de Odontología construyeran una forma innovadora de educación en salud bucal, basada en prácticas educativas y dinámicas, que contribuyó a visualizar un horizonte de desempeño más humanizado que supere a los antiguos. prácticas de atención puntual e inmediata.

Palabras clave: Odontología pediátrica, Promoción de la salud, Higiene bucal, Humanización de la atención.

\section{INTRODUÇÃO}

A educação em saúde bucal é passível de ser disponibilizada em qualquer espaço comunitário e até mesmo no ambiente familiar, escolar ou de trabalho (MONTE DO, et al., 2015). No entanto, a visita de rotina ao dentista não pode ser ignorada, tendo em vista que esse é o profissonal mais capacitado para relalizar o diagnóstico completo da condição bucal.

Assim, uma das maiores dificuldades encontradas tanto pelos pacientes quanto pelos dentistas é a presença de ansiedade e medo por parte dos indivíduos, principalmente quando se trata de crianças (GOES MPS, et al., 2010; MARTINS N e DIAS MR, 2016). O paciente infantil com elevado nível de ansiedade e estresse pode enxergar o cirurgião dentista, seu ambiente de trabalho e os instrumentais usados como uma ameaça ao seu bem-estar (CORTELO FM, et al., 2014).

Com isso, o atendimento torna-se mais difícil, demorado e traumático para ambos os lados sendo necessário que o dentista desenvolva uma abordagem de forma humanizada à esse público, algo que demonstre os benefícios do cuidado ao estado psicológico do paciente, e por isso os estudantes são treinados a conceber um ambiente de apoio para a criança, principalmente em contexto de ansiedade, medo e dor. Portanto, o trabalho com uso de técnicas da psicologia nesse cenário pode ajudar, fornecendo o bem-estar, para que as crianças se sintam capazes e fortes para enfrentar essas situações (LIMA KMA, et al., 2016).

Existe um consenso que a comunicação e o gerenciamento da mesma, dentre as várias técnicas da psicologia, se destaca por ser a base da construção de uma relação de amizade com o paciente infantil, e como o principal objetivo da comunicação é a compreensão, é imprescindível dispor de garantias de que a situação será menos ameaçadora possível, sendo assim, a comunicação e o condicionamento do paciente desde a sala de espera é de extrema importância, visto que o período pré-operatório é o que mais provoca ansiedade nas crianças (ALBUQUERQUE CM, et al. 2010).

Muitos trabalhos na literatura indicam que a educação em saúde bucal deve ter seu início, de preferência, ainda na fase pré-escolar da criança, pois é nessa etapa da vida delas que ocorre a aquisição de novas habilidades, conhecimentos, coordenação motora e de linguagem, aspectos estes que favorecem o melhor aprendizado e autonomia.

É importante ressaltar ainda que nesse período a criança demonstra grande interesse e capacidade de imitação de comportamentos, estando sujeita a reproduzir tanto hábitos benéficos quanto maléficos para sua saúde baseados em seus exemplos ou estímulos recebidos (GARBIN CAS, et al., 2014; LEITE GR, et al., 2015; SIGAUD CHS, et al., 2017). 
Ainda sobre a educação em saúde bucal com crianças na fase escolar os autores Dias MR, et al. (2015) relatam que é indispensável trabalhar o assunto buscando prevenir o aparecimento de cáries nessa faixa etária, pois é justamente no período dos cinco aos sete anos que a criança passa a adquirir a capacidade de escolha dos alimentos que compõem a sua dieta e desta maneira, construir hábitos mais saudáveis de alimentação de forma autônoma.

De acordo com Silva RBL, et al. (2017) é importante buscar instrumentos pedagógicos que possibilitem a troca de informações e a abertura do espaço para que os sujeitos envolvidos no contexto da educação infantil sintam-se capazes de estabelecer uma interação e um diálogo, proporcionando que o aprendizado seja algo atrativo, divertido e de boa valia para o indivíduo.

Araújo SM, et al. (2017) concluem em sua pesquisa que as atividades lúdicas são eficazes na motivação da higiene oral e contribuem para a redução do índice de placa, e ressaltam ainda que o emprego dessas atividades deve ser estimulado tanto no âmbito do sistema público de saúde quanto no privado.

Portanto, esse tipo de atividade pode ser vislumbrada como uma ferramenta facilitadora para compartilhar conhecimentos e também motivacional para as crianças, almejando atingir resultados muito melhores com relação à saúde bucal delas (FADEL CB, et al., 2015).

Com base no exposto, o presente artigo objetiva narrar a experiência vivida pelos acadêmicos do curso de Odontologia durante a realização das atividades do programa "Heróis do Sorriso", as quais foram voltadas para as crianças em acompanhamento odontológico da clínica escola da faculdade no intuito de melhorar o condicionamento, diminuir o medo e a ansiedade ao contato com o cirurgião-dentista e facilitar a realização dos tratamentos.

\section{RELATO DE EXPERIÊNCIA}

A Clínica Escola de Odontologia da Universidade Federal de Campina Grande (CEO-UFCG) é um órgão de prestação de serviços à saúde, de caráter público, que atende aos mais diversos grupos etários. Devido à oferta de disciplinas obrigatórias para conclusão do curso como a de Odontopediatria, a clínica também oferece os seus serviços de forma específica para esse público.

Foi baseado em evidências científicas como essas, que o eixo CEO-UFCG foi desenvolvido pelo programa de extensão "Heróis do Sorriso", objetivando prover o condicionamento dos pacientes de atendimento infantil no momento pré-operatório na sala de espera, e operatório na cadeira odontológica, usando brincadeiras e diálogos que pudessem fornecer o respaldo psicológico para um atendimento confortável para as crianças.

Todas as atividades a serem desenvolvidas eram planejadas com antecedência, e um cronograma era desenvolvido para uso mensal a partir de tema pré-determinado pelos discentes e docentes do programa. Materiais também eram confeccionados pelos integrantes visando sempre trazer o aspecto educativo juntamente com o lúdico. Vale a pena salientar que além das atividades focadas em temas de saúde bucal e geral, bem como em relação ao atendimento, os extensionistas também buscavam sempre iniciar as atividades com algo que fortalecesse a humanização e a criação de uma relação de confiança.

Os integrantes conviviam com a análise do comportamento e emoções, ao passo que, com a maioria dos pacientes mais antigos a relação já era bem estabelecida e podia-se desfrutar de um vínculo de confiança e tranquilidade, com outros também antigos e os novos, ainda se exigia o esforço de perceber e lidar com o medo e a ansiedade que eram demonstrados muitas vezes pela timidez, sudorese e resistência às brincadeiras.

Com o que já foi exposto, percebe-se que o foco de todas as intervenções eram a humanização e a empatia de tentar sentir e cumprir com as expectativas dos pacientes, bem como fortalecer a visão holística de estudantes e futuros profissionais de saúde, buscando sempre outros fatores e sinais que pudessem deduzir ou indicar que as crianças estavam sendo submetidas a maus tratos ou violência doméstica e sexual.

Como dito anteriormente, destaca-se dois momentos de contato dos extensionistas com as crianças, onde primeiramente serão descritas as atividades e a experiência do que era vivido no momento pré-operatório na sala de espera.

REAS/EJCH | Vol.12(11) | e4991 | DOI: https://doi.org/10.25248/reas.e4991.2020 Página 3 de 6 
Em geral, as ações na sala de espera seguiam a seguinte programação: momento "quebra-gelo", pequena palestra sobre o tema determinado e brincadeiras de cunho educativo e de recreação. No momento supracitado era realizado com os extensionistas indo até as crianças com algo que lhes interessasse como balões e desenhos, convidando-as a participar de atividades de descontração, ainda eram realizadas pinturas faciais de acordo com o que as crianças pedissem, e os discentes também disponibilizavam os seus rostos para que elas pudessem pintar.

As palestras nunca eram compostas por um monólogo, mas sim por uma união de diálogo, feito em linguagem adequada para o público, e uso de ferramentas lúdicas que pudessem auxiliar nessa comunicação, como: álbuns seriados, caixas surpresas, vídeos, músicas e jogos. Como já dito, os temas eram escolhidos para serem usados de forma mensal e variavam, dentre outros, em: cárie, dieta, traumatismo dentário, higiene pessoal, atendimento odontológico.

As brincadeiras de cunho educativo também eram feitas com materiais de apoio, comumente era realizada a dinâmica de amigos e inimigos dos dentes, por meio de jogo da memória com cartas confeccionadas em EVA, ou por meio do uso de caixas surpresas, onde a criança colocava a mão e retirava a ilustração de algum alimento ou produto e deveria informar se aquilo era bom ou ruim para os dentes, também era realizada a brincadeira da batata quente, onde eram feitas perguntas sobre o tema que havia sido tratado e sugeriam-se prendas como: mostrar como se usa o fio dental; como realizar a escovação e falar como eram realizados os procedimentos odontológicos e quais os benefícios do mesmo.

As brincadeiras de recreação variavam em amarelinha, morto ou vivo, pinturas, atividades com balões e tudo que as crianças sugerissem e fosse possível de se realizar, visto que esse era o momento delas e pelo fato dessa autonomia fornecer à criança a sensação de que tudo estava sob o seu controle, aumentando assim sua autoconfiança e consequentemente podendo proporcionar um período transoperatório satisfatório.

O segundo momento de contato dos extensionistas com as crianças, além do que já foi descrito anteriormente na sala de espera, era o acompanhamento durante a fase transoperatória, que visava oferecer à criança uma assistência integral, considerando o período de chegada até o momento que a criança concluía o seu atendimento.

A partir do condicionamento dos pacientes na sala de espera, os mesmos iam sendo chamados para o início dos seus respectivos procedimentos e com o intuito de manter a confiança que outrora a criança conquistou com os extensionistas, os mesmos acompanhavam a criança durante todo o procedimento odontológico, tendo em mente a utilização das técnicas de manejo comportamental em odontopediatria. A aplicação de técnicas não farmacológicas de controle do comportamento do paciente infantil, nos procedimentos odontológicos, é de extrema importância para o bom andamento dos mesmos, de maneira a proporcionar segurança à criança.

Com esse pensamento, os extensionistas auxiliavam os discentes que iriam realizar os procedimentos nos pacientes, nesse quesito a comunicação verbal e não-verbal, distração, a técnica dizer-mostrar-fazer e o reforço positivo foram meios utilizados inicialmente e durante todo o atendimento clínico para os pacientes que colaboravam com os procedimentos.

Essas técnicas citadas anteriormente sempre eram utilizadas com o intuito de manter o comportamento colaborativo da criança, engajando-a no seu próprio atendimento. Em alguns casos, quando o paciente não colaborava para a realização do procedimento, lançava-se mão do controle da voz, com a finalidade de chamar a atenção da criança para que a mesma entendesse que o procedimento precisava ser realizado.

Quando mesmo com o uso de todas essas técnicas de manejo, o paciente impedia que o procedimento fosse executado, a indicação da técnica de contenção física era admitida dentro do protocolo de atendimento, porém a mesma só era realizada quando existia a garantia da segurança do paciente e em última instância para os procedimentos que não poderiam ser adiados.

Com esse intuito, e utilizando esse protocolo, existia a garantia do bem-estar e da resolução dos problemas odontológicos dos pacientes. No entanto, dificilmente se fazia necessário o uso da contenção física, visto que o condicionamento realizado no momento pré-operatório ajudava a tornar o paciente colaborativo. 
Os alunos que eram responsáveis por atender e realizar os procedimentos clínicos, bem como os professores e orientadores da disciplina, sempre destacaram a mudança no perfil comportamental das crianças após o início do programa em questão, as mães sempre agradeciam e também se surpreendiam com a conduta dos pacientes frente aos procedimentos, e as próprias crianças se orgulhavam em falar que sua postura havia sido semelhante àquela ensinada pelos alunos extensionistas.

\section{DISCUSSÃO}

A prática de acolhimento realizada através do projeto de extensão que atua na Clínica-Escola de Odontologia da faculdade tem como foco principal fornecer informações para as crianças de forma lúdica, simples e criativa. Essa experiência visa incentivar o desenvolvimento do autocuidado com a cavidade bucal e familiarizar os pequenos com o ambiente clínico, para que se sintam menos aflitos quando forem atendidos, visto que ansiedade manifestada pelas crianças na consulta de odontopediatria é uma problemática com a qual os cirurgiões-dentistas se deparam frequentemente, constituindo uma relevante barreira no exercício clínico (MARTINS N e DIAS MR, 2016; SANTOS LGP,et al., 2020).

Na pesquisa realizada por Kronina L, et al. (2017), foram verificados fatores que poderiam relacionar a ansiedade em crianças com os tratamentos odontológicos. Eles utilizaram um questionário para que os pais avaliassem o nível de ansiedade dos filhos, seguido de uma avaliação do comportamento da criança no consultório.

Fatores como a presença dos pais, boas experiências em atendimentos anteriores e a explicação dos procedimentos e da importância deles, foram fatores que diminuíram a ansiedade dos pacientes. Muitos estudos afirmam que conquistar a confiança da criança a torna mais colaborativa no atendimento, por esse motivo, os alunos extensionistas tentavam conseguir essa confiança para que elas aderissem melhor aos tratamentos (FIDALGO TKS, et al., 2009; VALARELLI FP, et al., 2011).

Para Lima KMA, et al. (2016), a abordagem dos pacientes odontopediátricos não deve ser restrita apenas a cadeira de atendimento, o acolhimento deve ser iniciado ainda na sala de espera para que se possa compreender melhor o nível de ansiedade da criança e assim elaborar formas para tranquilizá-la e torná-la mais colaborativa, o que corrobora com a importância do projeto, visto que os extensionistas cumpriam a função de receber as crianças ainda na recepção e distraí-las com dinâmicas, brincadeiras e conversas na tentativa de tentar amenizar os medos e sanar as dúvidas dos jovens pacientes.

Durante $o$ acolhimento as crianças eram incentivadas a realizar os cuidados básicos de higiene oral, sendo também explicada a importância de uma alimentação adequada e os riscos que consumir alimentos cariogênicos em excesso pode ter. Para Cota ALS e Costa BJA (2017) e Negreiros PS, et al. (2018), a motivação em programas educativos-preventivos tem grande importância na conscientização de crianças e o uso de ferramentas que melhorem o entendimento e proporcionem uma boa transmissão de conhecimento são cruciais na educação de crianças, sendo ainda mais efetivas quando acompanhadas por sessões de reforço continuado.

Muitos pais também notaram melhorias no comportamento dos filhos e quando retornavam à clínica em outras consultas passavam a incentivar a participação deles nas atividades desenvolvidas pelos extensionistas.

Sendo os pais a figura de maior segurança que as crianças têm, ter o apoio dos mesmos é de grande importância não apenas durante os atendimentos odontológicos, mas também no incentivo à participação dessas atividades, posto que são eles os agentes de saúde familiar responsáveis pela motivação essencial para o tratamento odontopediátrico. Assim, os pais ou responsáveis devem ser encarados como aliados na promoção da saúde oral da criança (MACHADO MS, et al., 2009).

Por fim, constatou-se que o acolhimento proporcionado pelos acadêmicos de Odontologia que atuavam no projeto geravam uma melhoria bastante significativa no comportamento das crianças que eram atendidas, tal mudança foi notada principalmente pelos professores da disciplina de odontopediatria, que destacaram por diversas vezes a importância do projeto. 


\section{REFERÊNCIAS}

1. ALBUQUERQUE CM, et al. Principais técnicas de controle de comportamento em Odontopediatria. Arquivos em Odontologia, 2010; 46(2): 110-115.

2. ARAUJO SM, et al. Motivação de higiene bucal por meio de atividades lúdicas. Iniciação Científica Cesumar, 2017; 19(2): 111-117.

3. CORTELO FM, et al. Crianças em atendimento odontológico: arranjos psicológicos para a intervenção. Omnia Saúde, 2014; 11(1): 01-14.

4. COTA ALS, COSTA BJA. Atividades lúdicas como estratégia para a promoção da saúde bucal infantil. Saúde e Pesquisa, 2017; 10(2): 365-371.

5. DIAS MR, et al. Eu sou o Favolas: um instrumento de educação para a saúde em dentisteria. Journal of Human Growth and Development, 2015; 25(3): 325-330.

6. FADEL CB, et al. Gincana intelectual: instrumento de ação extensionista para educação em saúde. Revista Em Extensão, 2015; 14(1): 106-115.

7. FIDALGO TKS, et al. Percepção de alunos e profissionais da odontologia quanto a importância de um programa de extensão teórico- prático em odontopediatria.Rev. Saúde Com, 2009; 5(2): 90-96.

8. GARBIN CAS, et al. Conhecimento sobre saúde bucal e práticas desenvolvidas por professores do ensino fundamental e médio. RFO, 2014; 18(3): 321-327.

9. GOES MPS, et al. Ansiedade, medo e sinais vitais dos pacientes infantis. Revista Odontologia Clínico- Científica, 2010; 9(1): 39-44.

10. KRONINA L, et al. Psychosocial factors correlated with children's dental anxiety. Stomatologija, Baltic Dental and Maxillofacial Journal, 2017; 19(3): 84-90.

11. LEITE GR, et al. Saúde bucal na educação infantil, responsabilidade de quem? Itinerarius Reflectionis, 2015; 11(1).

12. LIMA KMA, et al. Psicologia e odontopediatria: possibilidade de atuação em uma clínica - escola. Revista Expressão Católica Saúde, 2016; 1(1): 1-19.

13. MACHADO MS, et al. Participação dos pais na toma da de decisões no atendimento odontológico de seus filhos. Revista de Odontologia da Universidade Cidade de São Paulo, 2009; 21(1): 38-47.

14. MARTINS N, DIAS MR. Contágio emocional de ansiedade encarregado de educação criança em odontopediatria. Revista Portuguesa de Estomatologia, Medicina Dentária e Cirurgia Maxilofacial, 2016; 57(3): 164-170.

15. MONTE DO, et al. Conscientização da Higienização bucal na população brasileira. Ciencias Biologicas e da Saúde, 2015; 2(2): 53-60.

16. NEGREIROS PS, et al. Perfil epidemiológico da cárie dentária em pré-escolares de uma escola pública do município de Manaus-AM. Arquivos de Ciências da Saúde da UNIPAR, 2018, 22(1): 11-15

17. SANTOS LGP, et al. Manejo do comportamento infantil e saúde bucal na sala de espera: um relato de experiência. Research, Society and Development, 2020, 9(7): e888975069.

18. SIGAUD CHS, et al. Promoting oral care in the preschool child: effects of a playful learning intervention. Revista Brasileira de Enfermagem, 2017; 70(3): 519-525.

19. SILVA AM, COSTA HS. Estágio no Departamento de Odontopediatria da Universidade da Flórida: um relato de experiência. Revista da ABENO, 2018; 18(1): 93-102.

20. SILVA RBL, et al. A roda da conversa na educação infantil: instrumento de silenciamento ou amplificação da voz da criança? Revista Eletrônica de Educação, 2017; 11(3): 1001-1019.

21. VALARELLI FP, et al. Importância dos programas de educação e motivação para saúde bucal em escolas: relato de experiência. Revista Odontologia Clínico- Científica, 2011, 10(2): 173-176. 